\section{Neues Botulinumtoxin-Präparat für die Glabellafalte}

\begin{abstract}
— Die Palette der Botulinumtoxin-Präparate in der ästhetischen Medizin ist um eine Option breiter geworden. Das Unternehmen Merz bringt mit Bocouture ${ }^{\circledR}$ ein Botulinumtoxin-Typ-A auf den Markt, das auf komplexbildende Proteine verzichtet.

Das neue Präparat, das für die Therapie bei Glabellafalten zugelassen ist, wurde in einer randomisierten Phase-III-Studie bei 381 Patienten mit konventionellen BotulinumtoxinPräparaten (Botox ${ }^{\circledR}$, Vistabel ${ }^{\circledR}$ ) verglichen. Primärer Endpunkt war das Therapieansprechen nach vier Wochen, definiert als Verbesserung auf der Facial-Wrinkle-Skala (FWS) von mindestens einem Punkt. Die Bewertung erfolgte durch behandelnde Ärzte, Patienten und unabhängige, hinsichtlich der Therapie geblindete Experten, die den Therapieerfolg nur anhand der Bilder zu bewerten hatten.

„Die Ergebnisse waren für beide Gruppen praktisch identisch, sodass der angestrebte
\end{abstract}

Beleg für die Nicht-Unterlegenheit erbracht werden konnte“, sagte Dr. Gerhard Sattler, Darmstadt. Die durch die unabhängigen Experten ermittelten Ansprechraten lagen bei 96,4\% für das neue Präparate und 95,7\% für die konventionellen Präparate.

„Der Vorteil der neuen Formulierung ist, dass sie ganz ohne komplexbildende Proteine auskommt", so Sattler. Dies könne sich positiv auf die Antigenität des Präparats auswirken. Als günstig bewertete Sattler auch die vergleichsweise geringe Diffusion von Bocouture ${ }^{\circledR}$. Dadurch sei eine sehr gezielte Applikation möglich, wie sie gerade im Gesicht oft erforderlich ist, um kosmetisch gute Resultate zu erzielen.

gvg

Symposium: Pure performance: Introducing a new way to look as vibrant as you feel. Im Rahmen der 18. EADV-Tagung, Berlin, 9.10.2009 (Veranstalter: Merz Aesthetics).

\title{
TNFalpha-Blocker für drei Indikationen
}

- Für die Therapie entzündlich-rheumatischer Erkrankungen sind derzeit sieben Biologika zugelassen, darunter zwei voll humanisierte monoklonale Antikörper, deren Wirkprinzip auf der Blockade des proinflammatorischen Zytokins TNFalpha beruht. Seit kurzem ist das Spektrum der verfügbaren TNFalpha-Inhibitoren um den neuen humanen monoklonalen Antikörper Golimumab (Simponi ${ }^{\circledR}$ ) erweitert, der sich für den Patienten als deutlicher Fortschritt erweisen wird, betonte Prof. Klaus Krüger, München. Diese Substanz ist als erste ihrer Art ab dem Tag der Einführung für die drei Indikationen rheumatoide Arthritis (RA), ankylosierende Spondylitis (AS) und Psoriasis-Arthritis (PA) verfügbar. Grundlage für diese Dreifach-Zulassung sind die positiven Ergebnisse von fünf parallel durchgeführten plazebokontrollierten Phase-III-Studien mit insgesamt 2.303 Teilnehmern.

Die Wirksamkeitsdaten belegen, dass mit Golimumab ein Biologikum für die Behandlung der genannten drei Formen entzündlich-rheumatoider Erkrankungen zur Verfügung steht, dessen Effektivität mit der anderer TNFalpha-Blocker vergleichbar ist, erklärte Prof. Jürgen Wollenhaupt, Hamburg. Als großen Vorteil bezeichnete er die monatlich einmalige subkutane Injektion des Wirkstoffs, die vom Patienten komfortabel selbst per Autoinjektor mit fixer Wirkstoffkonzentration durchgeführt werden kann. Dies komme vor allem Rheumapatienten mit Bewegungsstörungen und eingeschränkter Handmotorik entgegen.

Den Zulassungsstudien zufolge verbessert das Biologikum signifikant die Gelenksymptome und Beweglichkeit bei AS- und den Hautzustand bei PA-Patienten. Golimumab erwies sich als hochwirksam bei Patienten, die erfolglos mit Methotrexat oder einem anderen TNFalpha-Blocker vorbehandelt wurden, betonte Wollenhaupt. Das Nebenwirkungsprofil entspreche dem von Plazebo mit Ausnahme möglicher temporärer Irritationen an der Injektionsstelle.

Launch-Pressekonferenz „Simponi ${ }^{\circledR}$ (Golimumab“) - eine neue Dimension in der AntiTNFalpha-Therapie. München, 10.7.2009 (Veranstalter: Essex Pharma).
Ästhetische Narben mit Leukosan ${ }^{\circledR}$ Wundnahtstreifen

Narben lassen sich nach Operationen nicht vermeiden, wünschenswert ist aber ein ästhetisches Gesamtergebnis. Die optimierten Wundnahtstreifen Leukosan ${ }^{\circledR}$ haben eine zuverlässige Klebkraft und ermöglichen eine spannungsfreie Wundrandadaption. Die einfachere Applikation erleichtert das sterile und hygienische Arbeiten und reduziert das Infektionsrisiko. Wundnahtstreifen dienen zum atraumatischen und nahtfreien Verschluss kleiner Wunden und Inzisionen, als Ergänzung zur Subkutan- oder Intrakutannaht oder zur postoperativen Versorgung nach Klammerentfernung. Die Strips lassen sich schmerzlos und ohne Rückstände nach fünf bis sieben Tagen entfernen.

Nach Information von BSN medical

Kreussler Young Scientists' Sclerotherapy Award 2009 Erstmalig wurde der Forschungs-Förderungspreis der Union Internationale de Phlebologie (UIP), der „Kreussler Young Scientists' Sclerotherapy Award“, verliehen. Der von Kreussler gestiftete, mit insgesamt 10.000 Euro dotierte Preis soll, ,junge Nachwuchswissenschaftler bei klinischen, präklinischen oder experimentellen Forschungsvorhaben zur Sklerotherapie von Krampfadern unterstützen und sie zum wissenschaftlichen Arbeiten animieren", fasste Prof. Eberhard Rabe, Präsident der UIP und der Deutschen Gesellschaft für Phlebologie, den Grundgedanken der auf zwei Jahre angelegten Förderung zusammen. Erste Preisträgerin ist Nina Tetsch von der Hautklinik der Universität Lübeck. Sie hatte gemeinsam mit ihrer Arbeitsgruppen-Leiterin, Dr. Birgit Kahle, die jetzt prämierte Idee zur Untersuchung einer Verödungstherapie für funktionsschwache Stammvenen mit Hilfe von aufgeschäumten Verödungsmitteln. Dabei wird der Effekt der TumeszenzLokalanästhesie, getestet.Die Ergebnisse der Studie werden beim nächsten UIP-Kongress 2011 in Prag präsentiert. Informationen zum 2nd UIP Research Fellowship - Kreussler Young Scientists' Sclerotherapy Award 2011 können unter award@kreussler.com angefordert werden.

Nach Information von Kreussler 